\title{
Influence of cyclonic and anti-cyclonic eddies on plankton biomass, activity and diversity in the southeastern Mediterranean Sea \\ Supplementary Material
}
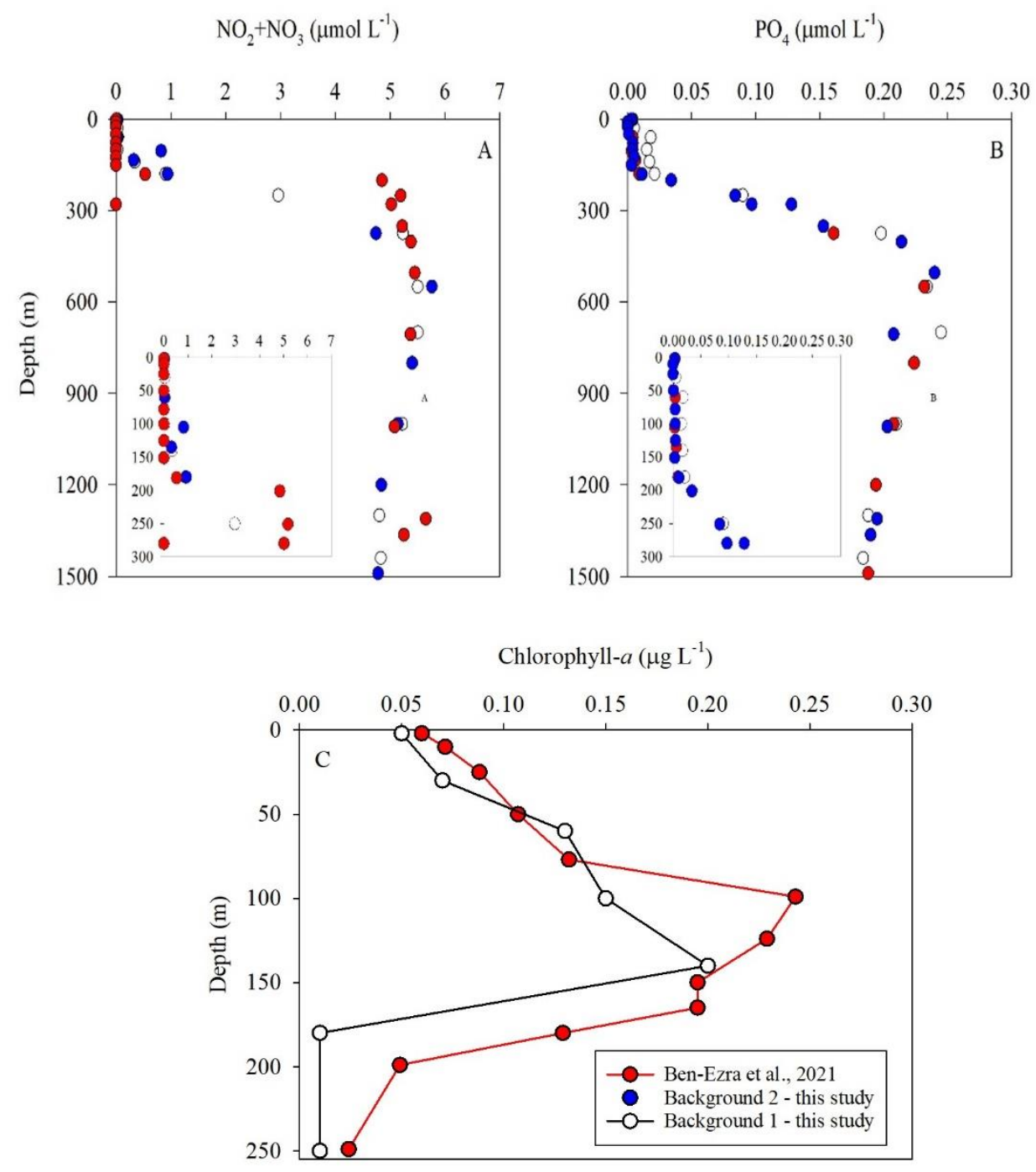

Figure $\mathrm{S1}$ - Comparison between the vertical profiles of $\mathrm{NO}_{2}+\mathrm{NO}_{3}(\mathrm{~A}), \mathrm{PO}_{4}(\mathrm{~B})$ and chlorophyll- $a(\mathrm{C})$ at the station used as 'background' in this study (white, Lat. $32.95 \mathrm{~N}$, Lon. 34.46 E); a station located between the eddies (blue, Lat. 32.66; Lon. 33.76) and an offshore station at the THEMO mooring (red, Lat. 32.82; Lon. 34.95, Ben-Ezra et al., 2021). All offshore stations were sampled at the same time as in our study (October 2018). 
Kruskal-Wallis One Way Analysis of Variance on Ranks shows the vertical profiles were insignificantly different; $\mathrm{P}=0.63, \mathrm{P}=0.43$ and $\mathrm{P}=0.24$ for $\mathrm{NO}_{2}+\mathrm{NO}_{3}, \mathrm{PO}_{4}$ and chlorophyll$a$, respectively. Insert in panels A and B: the upper $300 \mathrm{~m}$.

15
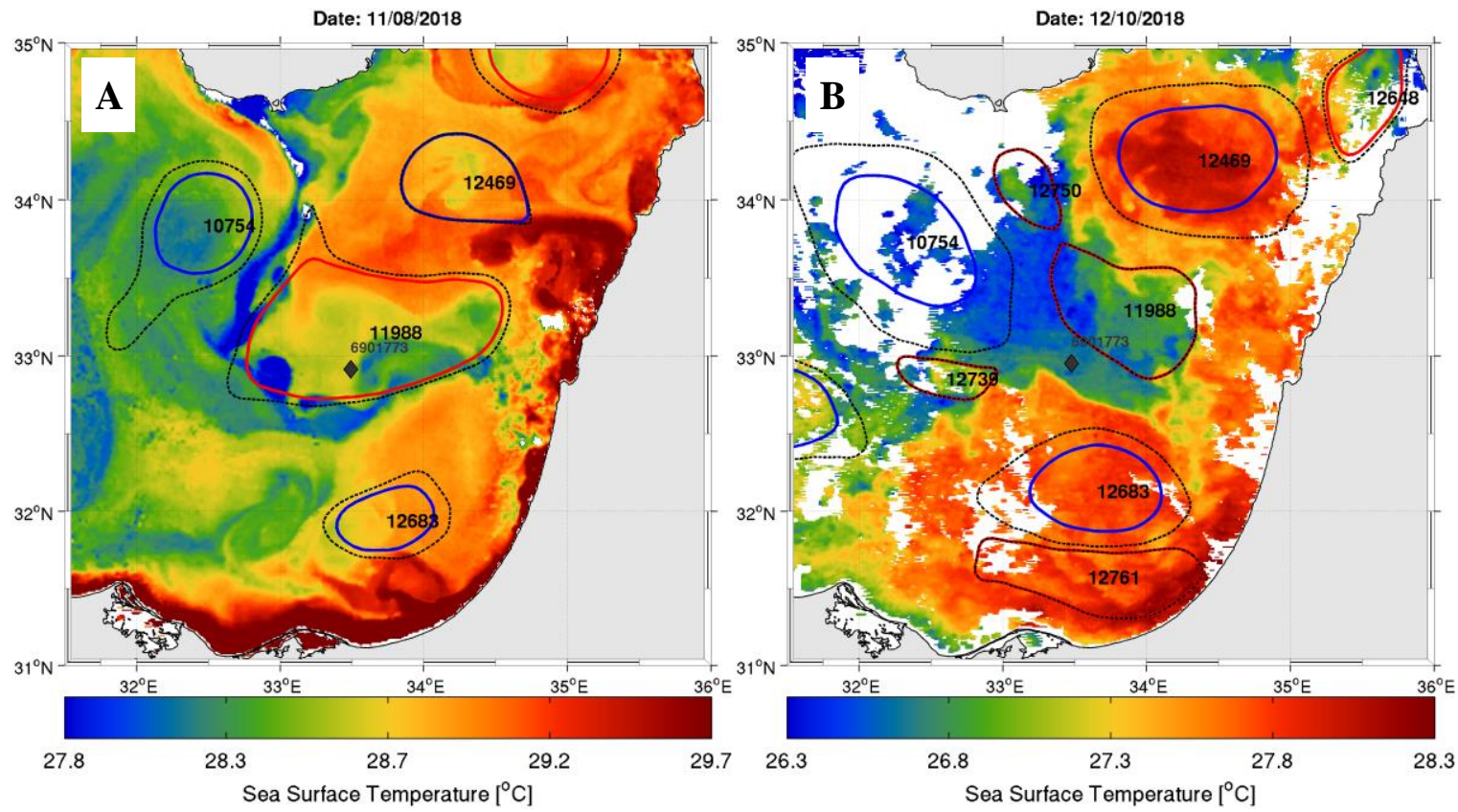

Figure S2 - Sea surface temperature maps with eddies detected by the AMEDA algorithm for $11 / 08 / 2018$ (A), and 12/10/2018 (B). 


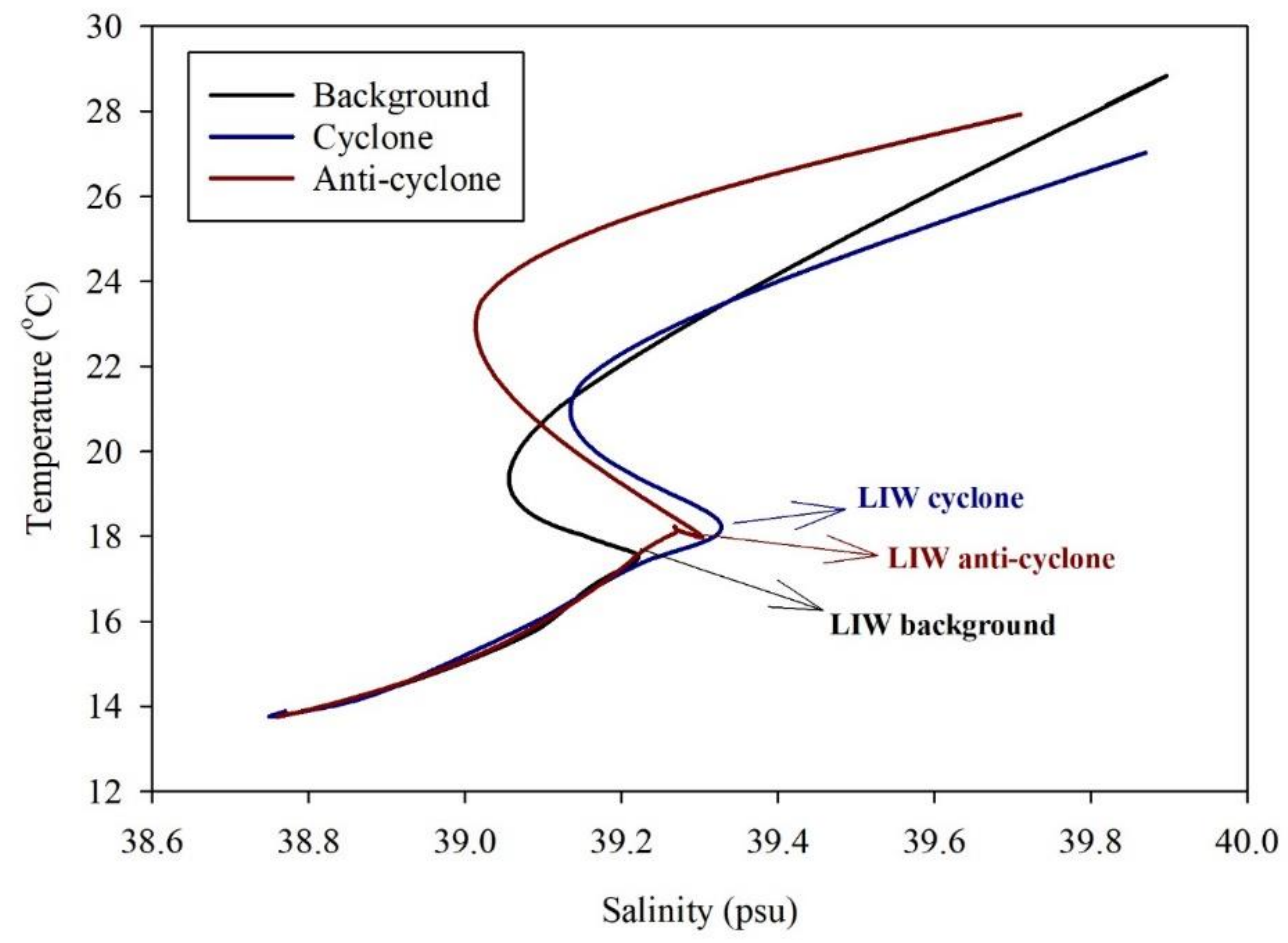

Figure S3 - A temperature-salinity (T-S) diagram of the stations sampled and the location of the LIW in each vertical profile. The black line signifies the background station, the red line the anti-cyclone and the blue line the cyclone. 
Proteobacteria; Gammaproteobacteria; SAR86 clade Bacteroidota; Bacteroidia; Flavobacteriales Cyanobacteria; Cyanobacteriia; Synechococcales SAR324 clade (Marine group B)

Proteobacteria; Alphaproteobacteria; Rhodospirillales Verrucomicrobiota; Verrucomicrobiae; Opitutales

Proteobacteria; Gammaproteobacteria; Cellvibrionales Proteobacteria; Alphaproteobacteria; Puniceispirillales Proteobacteria; Alphaproteobacteria; Rhodobacterales Proteobacteria; Gammaproteobacteria; UBA10353 marine group Proteobacteria; Alphaproteobacteria; Rickettsiales Proteobacteria; Alphaproteobacteria; Defluviicoccales Planctomycetota; Planctomycetes; Pirellulales NB1-j order

Proteobacteria; Gammaproteobacteria; Oceanospirillales Gemmatimonadota; BD2-11 terrestrial group order Acidobacteriota; Vicinamibacteria; Vicinamibacterales Actinobacteriota; Acidimicrobiia; Microtrichales Thermoplasmatota; Thermoplasmata; Thermoplasmata Verrucomicrobiota; Verrucomicrobiae; Arctic97B-4 marine group Planctomycetota; Phycisphaerae; Phycisphaerales Planctomycetota; OM190; OM190

Thermoplasmatota; Thermoplasmata; Marine Group II Proteobacteria; Alphaproteobacteria; Parvibaculales Actinobacteriota; Acidimicrobiia; Actinomarinales Proteobacteria; Gammaproteobacteria; KI89A_clade Marinimicrobia (SAR406 clade) Crenarchaeota; Nitrososphaeria; Nitrosopumilales Chloroflexi; Dehalococcoidia; SAR202 clade Proteobacteria; Alphaproteobacteria; SAR11 clade

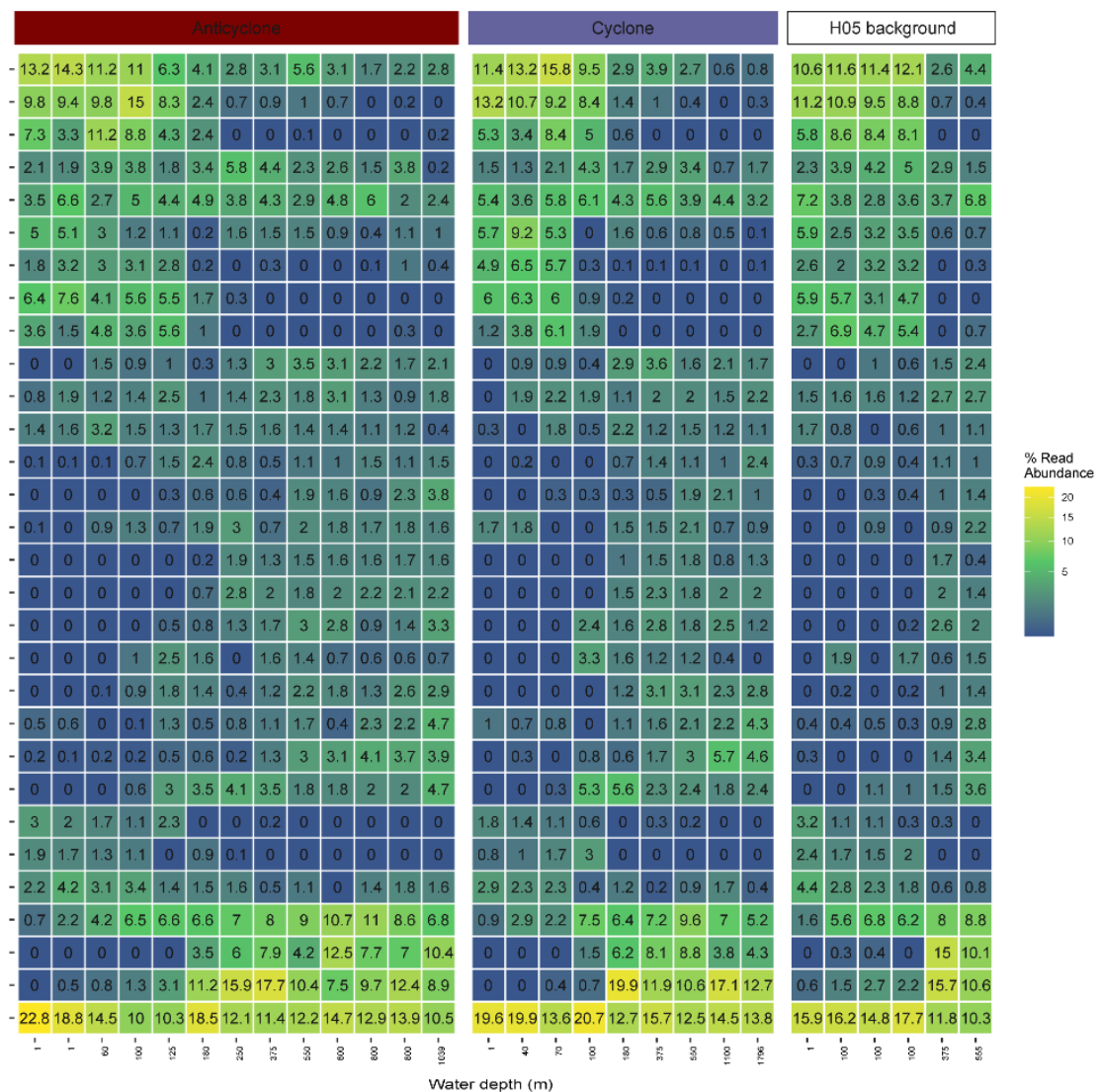

Water depth $(\mathrm{m})$

Figure S4. The relative abundance of 30 most-abundant bacterial and archaeal lineages (order level) was collected at cyclonic and anti-cyclonic eddies, and an uninfluenced background station (H05) at the southeastern Mediterranean Sea during October 2018, as stations are shown. 


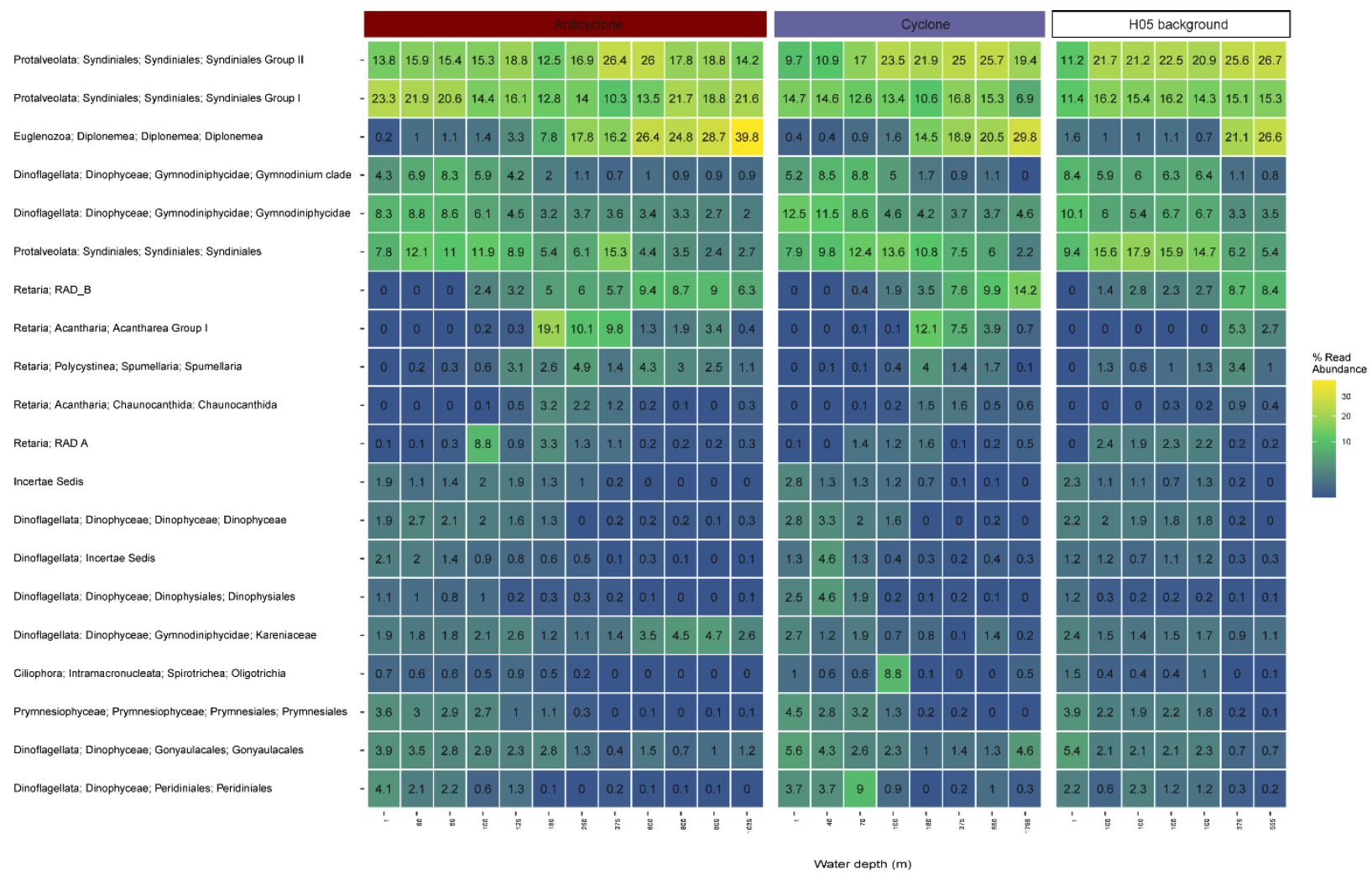

Figure S5. The relative abundance of 20 most-abundant unicellular eukaryotic lineages (taxonomy level 5 or the highest detectable level below the 5th) collected at cyclonic and 35 anti-cyclonic eddies, and an uninfluenced background station (H05) at the southeastern Mediterranean Sea during October 2018, as estimated by read abundance. Results of replicate casts in anti-cyclone and control H05 stations are shown. 

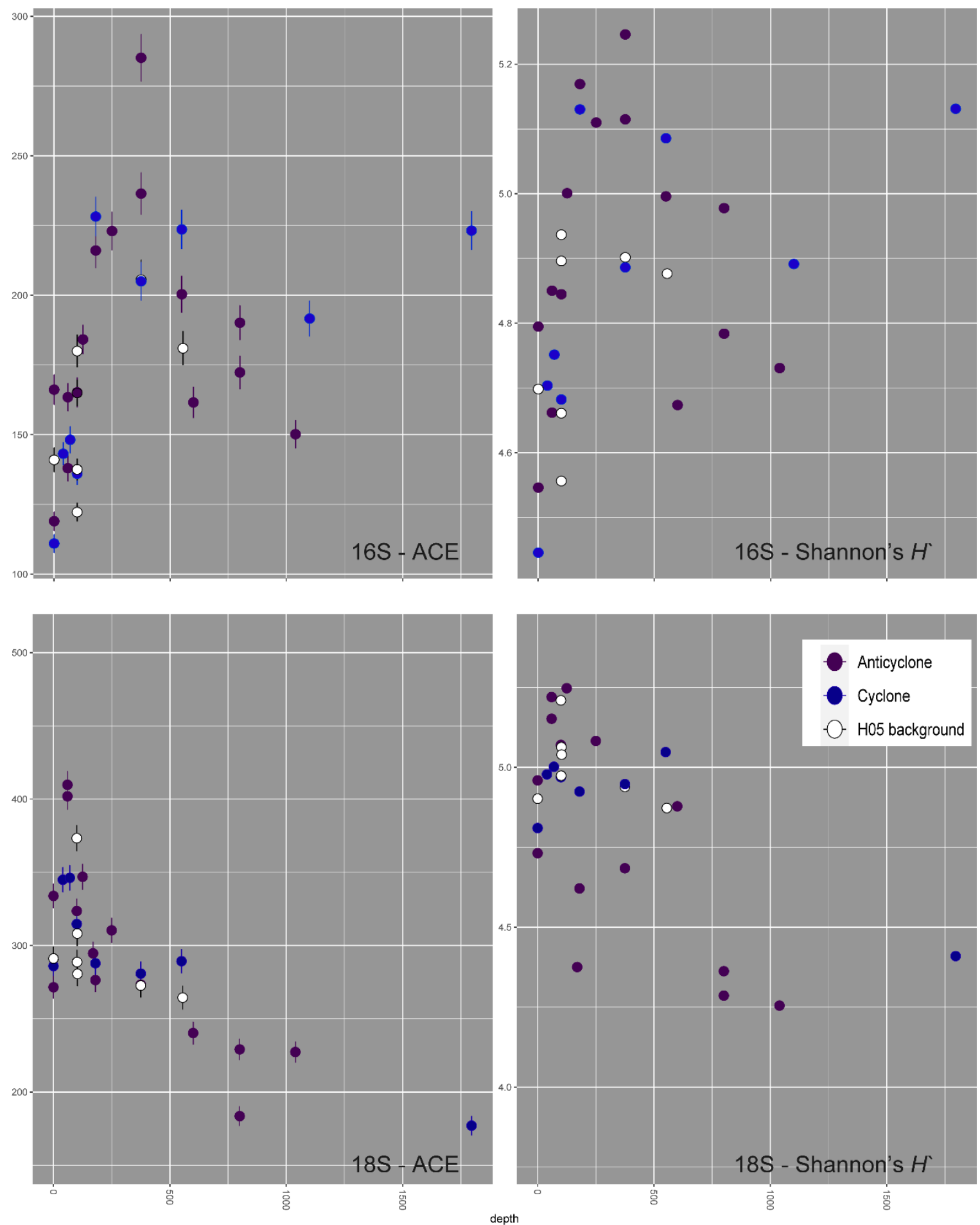

Figure S6. Species richness (as ACE) and diversity (as Shannon's $H^{\prime}$, log 10 base) in bacterioplankton (top) and unicellular planktonic eukaryotes (bottom) communities, estimated by amplicon sequence variant counts. Eukaryotic diversity decreases with depth, while that of bacterioplankton peaks below the deep chlorophyll maximum. 


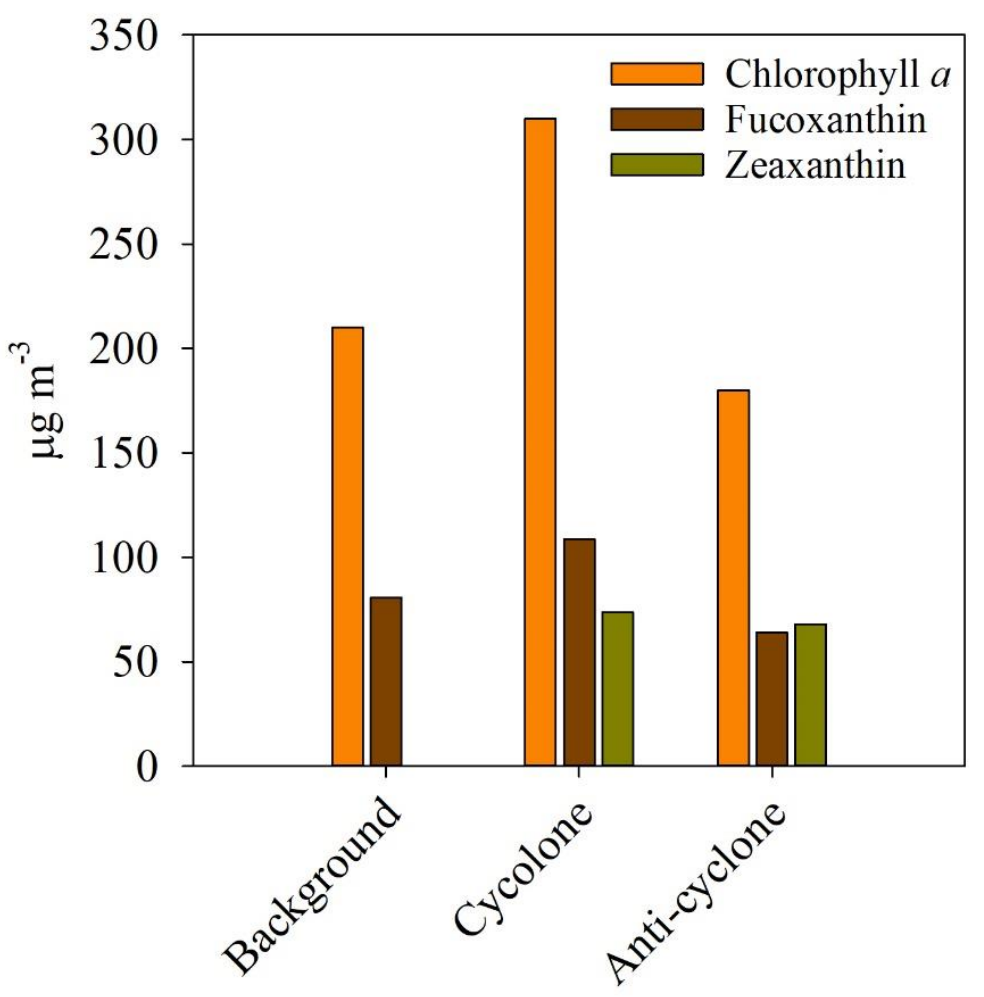

45 Figure S7. Main photosynthetic pigment markers that were found at the DCM at each station. Chlorophyll $a$ - the main photosynthetic pigment; Fucoxanthin - auxiliary pigment of diatoms, chrysophytes and some prymnesiophytes; and Zeaxanthin - auxiliary pigment marker of green algae and cyanobacteria. 


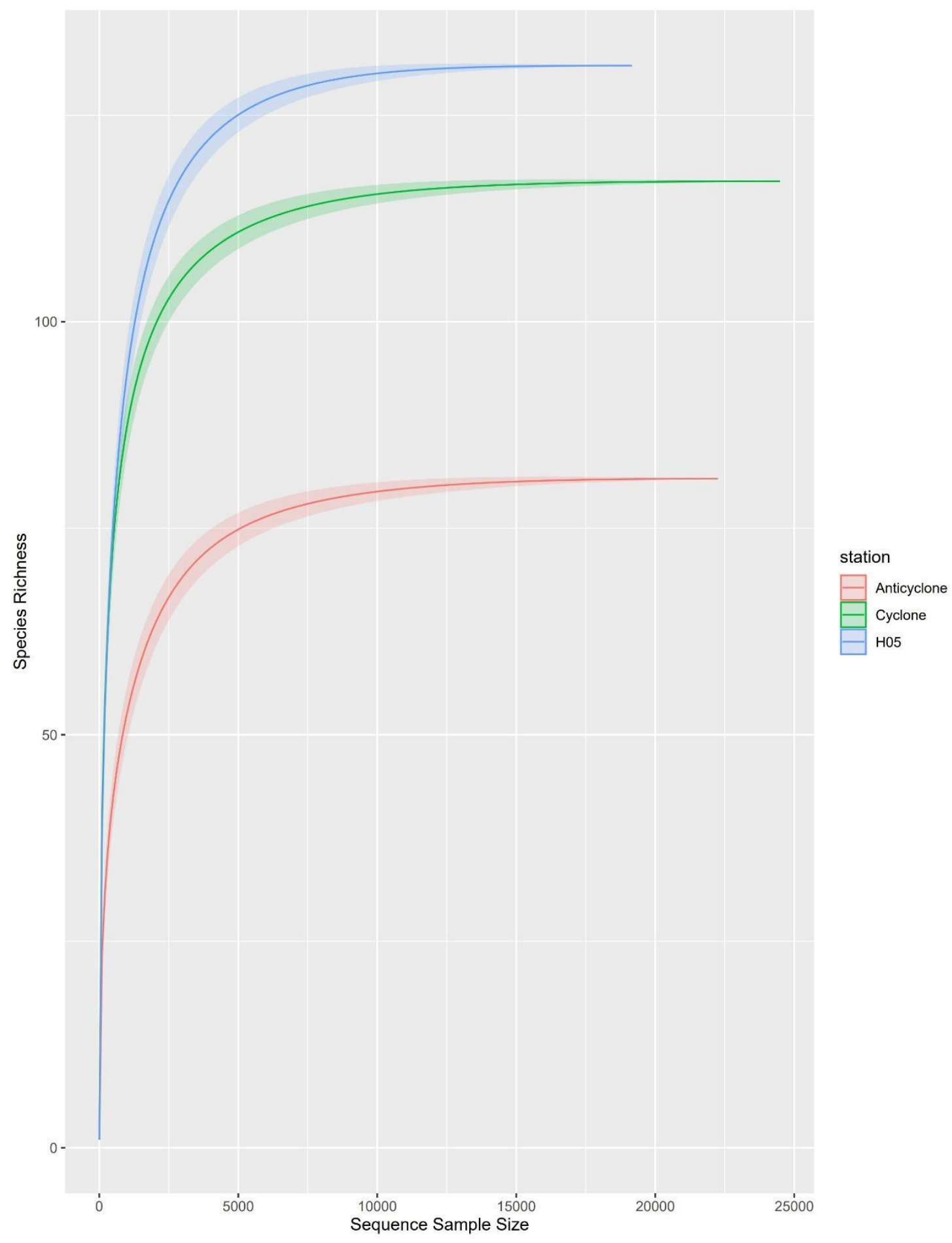

50 Figure S8. Rarefaction curves of observed 18S rRNA gene ASVs retrieved from 0-300m vertical net samples of mesozooplankton $>100-\mu \mathrm{m}$ collected at cyclonic and anti-cyclonic eddies and an uninfluenced background station (H05) at the Eastern Mediterranean Sea during October 2018. 


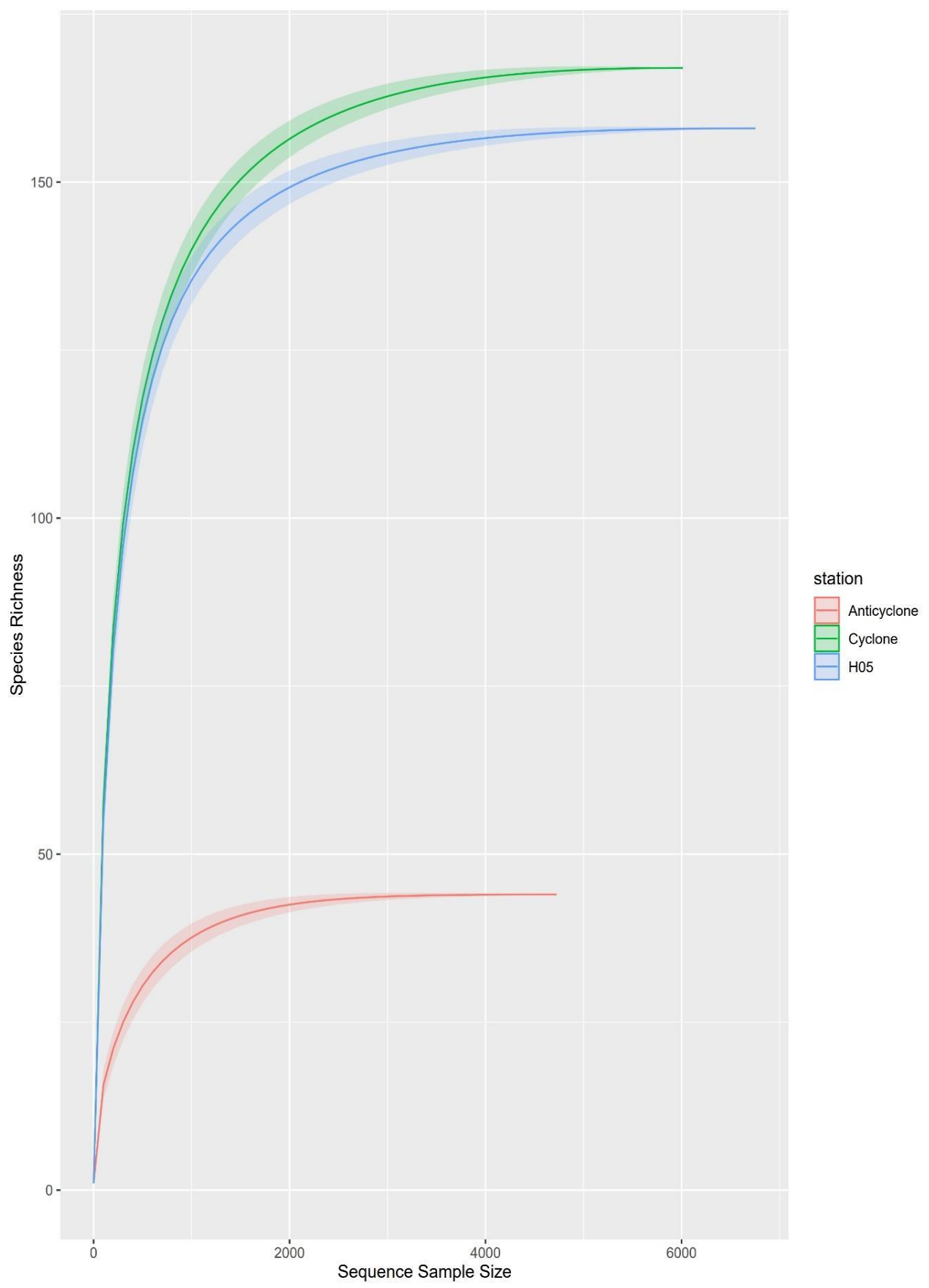

55

Figure S9. Rarefaction curves of observed COI gene ASVs retrieved from 0-300m vertical net samples of mesozooplankton $>100-\mu \mathrm{m}$ collected at cyclonic and anti-cyclonic eddies, and an uninfluenced background station (H05) at the Eastern Mediterranean Sea during October 2018. 


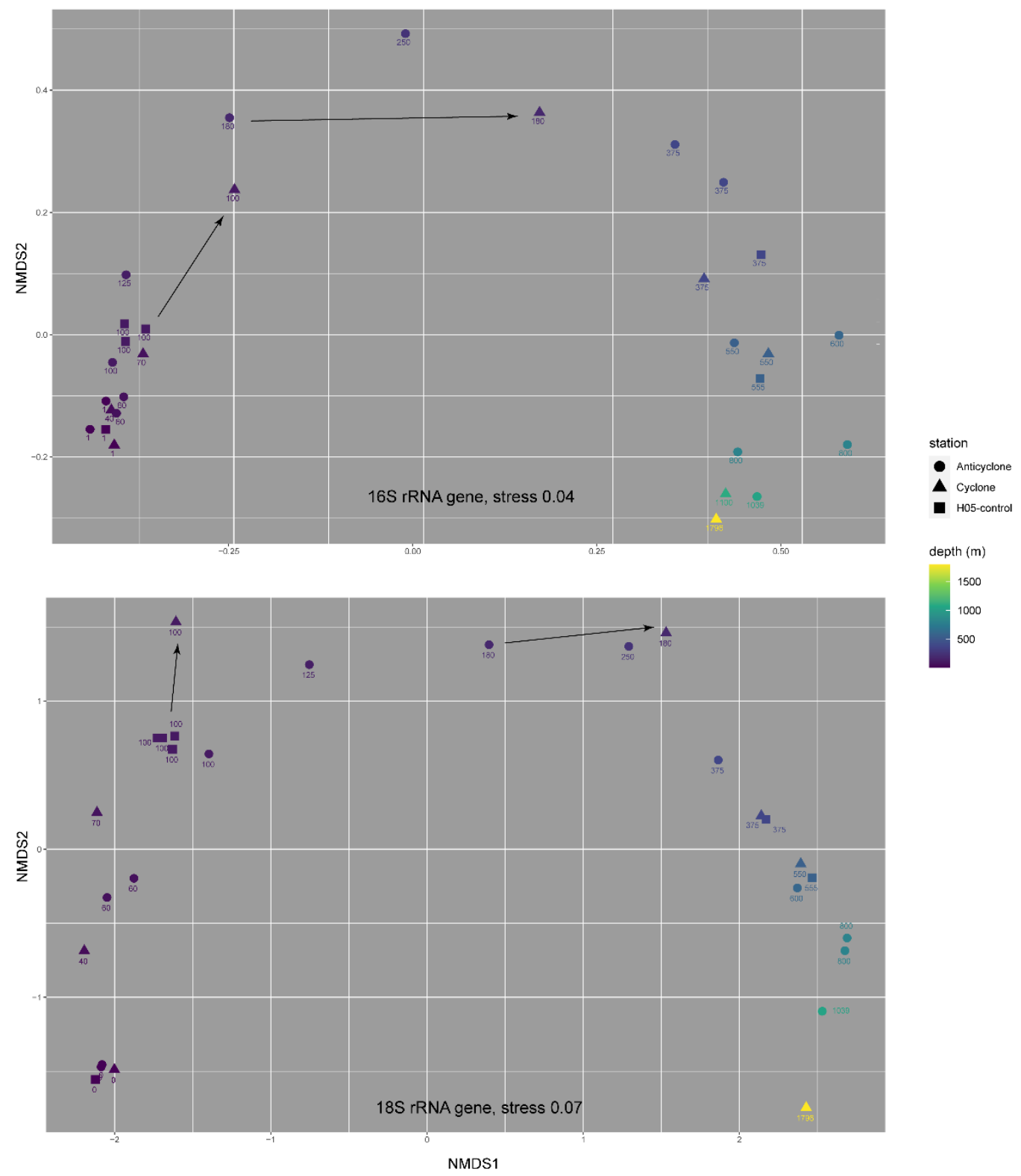

Figure S10. Non-metric multidimensional scaling (NMDS, based on the Bray-Curtis dissimilarity in amplicon sequence variants) plots, showing a potential shift in the composition of bacterioplankton (top) and unicellular planktonic eukaryotes (bottom) 65 communities at the deep chlorophyll maximum and $180 \mathrm{~m}$ depths of the cyclonic station, in comparison to the respective depths in the background (H05) and anticyclonic stations. The numbers next to symbols are depth (m). 
Table S1 - Results of statistical comparison (one-way ANOVA and Bonferroni-corrected post-hoc t-test, $\mathrm{P}<0.05)$ between variables $(0-180 \mathrm{~m})$ at the different stations. The letters represent the different statistical groups. N.A - Not applicable.

\begin{tabular}{|c|c|c|c|}
\hline Variable & Background & Cyclone & Anti-cyclone \\
\hline NOx $\left(m m o l ~ m^{-2}\right)$ & $\mathrm{B}$ & A & $\mathrm{C}$ \\
\hline $\mathrm{PO}_{4}\left(\mathrm{mmol} \mathrm{m}^{-2}\right)$ & A & A & $\mathrm{B}$ \\
\hline $\mathrm{Si}(\mathrm{OH})_{4}\left(\mathrm{mmol} \mathrm{m}{ }^{-2}\right)$ & A & $\mathrm{B}$ & A \\
\hline Chl- $a\left(\mathrm{mg} \mathrm{m}^{-2}\right)$ & A & A & $\mathrm{B}$ \\
\hline Synechococcus $\left(\times 10^{10}\right.$ cells $\left.\mathrm{m}^{-2}\right)$ & A & $\mathrm{C}$ & $\mathrm{B}$ \\
\hline Prochlorococcus $\left(\times 10^{10}\right.$ cells $\left.\mathrm{m}^{-2}\right)$ & $\mathrm{B}$ & A & $\mathrm{C}$ \\
\hline Pico-eukaryotes $\left(\mathrm{x} 10^{10}\right.$ cells $\left.\mathrm{m}^{-2}\right)$ & A & $\mathrm{B}$ & A \\
\hline Nano-eukaryotes $\left(\mathrm{x} 10^{10}\right.$ cells $\left.\mathrm{m}^{-2}\right)$ & A & $\mathrm{B}$ & $\mathrm{A}$ \\
\hline Total pico/nano-phytoplankton biomass $\left(\mathrm{mg} \mathrm{m}^{-2}\right.$ ) & $\mathrm{B}$ & $\mathrm{C}$ & $\mathrm{A}$ \\
\hline Heterotrophic bacteria $\left(\mathrm{x} 10^{10} \mathrm{cells} \mathrm{m}^{-2}\right)$ & A & B & $\mathrm{C}$ \\
\hline Heterotrophic bacteria biomass $\left(\mathrm{mg} \mathrm{m}^{-2}\right)$ & A & B & $\mathrm{C}$ \\
\hline Zooplankton ( $\mathrm{mg} \mathrm{m}^{-2}$ ) & N.A & N.A & N.A \\
\hline Zooplankton / total phytoplankton biomass & N.A & N.A & N.A \\
\hline $\mathrm{PP}\left(\mathrm{gC} \mathrm{m}^{-2} \mathrm{~d}^{-1}\right)$ & $\mathrm{B}$ & A & $\mathrm{B}$ \\
\hline $\mathrm{AN}-\mathrm{PP} / \mathrm{Chl} \cdot a\left(\mathrm{gC} \mathrm{gChl} \cdot a^{-1} \mathrm{day}^{-1}\right)$ & $\mathrm{B}$ & A & $\mathrm{B}$ \\
\hline $\mathrm{BP}\left(\mathrm{gC} \mathrm{m}^{-2} \mathrm{~d}^{-1}\right)$ & A & $\mathrm{B}$ & A \\
\hline $\mathrm{BP} / \mathrm{BA}\left(\mathrm{x} 10^{14} \mathrm{gC}\right.$ cell $\left.^{-1} \mathrm{day}^{-1}\right)$ & $\mathrm{B}$ & $\mathrm{C}$ & A \\
\hline $\mathrm{BP} / \mathrm{PP}$ & A & B & A \\
\hline
\end{tabular}

